\title{
Effects of lifestyle physical activity on perceived symptoms and physical function in adults with fibromyalgia: results of a randomized trial
}

\author{
Kevin R Fontaine ${ }^{1 *}$, Lora Conn ${ }^{1}$, Daniel J Clauw²
}

\begin{abstract}
Introduction: Although exercise is therapeutic for adults with fibromyalgia (FM), its symptoms often create obstacles that discourage exercise. We evaluated the effects of accumulating at least 30 minutes of self-selected lifestyle physical activity (LPA) on perceived physical function, pain, fatigue, body mass index, depression, tenderness, and the six-minute walk test in adults with FM.

Methods: Eighty-four minimally active adults with FM were randomized to either LPA or a FM education control (FME) group. LPA participants worked toward accumulating 30 minutes of self-selected moderate-intensity LPA, five to seven days per week, while the FME participants received information and support.

Results: Seventy-three of the 84 participants (87\%) completed the 12-week trial. The LPA group increased their average daily steps by 54\%. Compared to FME, the LPA group reported significantly less perceived functional deficits $(P=.032)$ and less pain $(P=.006)$. There were no differences between the groups on the six-minute walk test $(P=.067)$, fatigue, depression, body mass index, or tenderness.
\end{abstract}

Conclusions: Accumulating 30 minutes of LPA throughout the day produces clinically relevant changes in perceived physical function and pain in previously minimally active adults with FM.

Trial Registration: clinicaltrials.gov NCT00383084

\section{Introduction}

Fibromyalgia (FM) is a chronic, multidimensional disorder characterized by persistent, widespread body pain and tenderness [1]. FM is estimated to occur in $2 \%$ of the U.S. general population, affecting about eight times more women than men [2,3]. Symptoms associated with FM include body pain, fatigue, sleep disruption, headache, memory or concentration problems, mood disturbances, and irritable bowel syndrome [4]. FM often substantially hampers day-to-day functioning and is a primary cause of disability [5].

Even with the recent Food and Drug Administration approval of medications to treat FM, pharmacotherapy generally produces modest and inconsistent benefits on symptoms, functioning, and quality of life [6]. As such, nonpharmacologic treatments, such as exercise and

\footnotetext{
* Correspondence: kfontai1@jhmi.edu

'Division of Rheumatology, Johns Hopkins University School of Medicine, 5200 Eastern Avenue, Baltimore, MD 21224, USA
}

cognitive-behavioral interventions, are recommended to assist people with FM to better manage the array of symptoms and functional deficits [6]. Although exercise has been shown to be beneficial [for example, [7]], the symptoms of FM often create obstacles that deter many from exercising consistently enough to derive benefits [8]. Thus, finding new ways to promote increased physical activity in persons with FM that can be sustained overtime is important.

One promising approach is to ask people with FM to increase their lifestyle physical activity (LPA). LPA involves working toward meeting the U.S. Surgeon General's 1996 Physical Activity Recommendations of accumulating at least 30 minutes, above one's usual activity, of moderate-intensity physical activity five to seven days a week by integrating short bouts of activity into the day, such as increasing the amount of walking, performing more yard work, using the stairs and so on [9-11]. Although it is unclear whether a continuous 30 minute 
bout of physical activity is superior to accumulating smaller (10- to 15-minute) bouts of activity with regard to health outcomes, asking people with FM to accumulate small bouts of physical activity throughout the day, as opposed to being active for 30 consecutive minutes, might be less taxing and therefore easier to initiate and sustain over time. In a pilot study [12], we found that small bouts of LPA promoted a 70\% increase in physical activity in FM. However, in that small study LPA did not produce significant benefits on pain, fatigue, or perceived physical function compared to controls.

As part of an ongoing randomized trial designed to investigate the effects of LPA on ambulatory reports of physical activity, pain and fatigue, as well as measures of fitness, pain threshold and pain tolerance, we also collected questionnaire-based data on these variables. This paper presents the results on questionnaire-based assessments of perceived physical function, pain, fatigue and depression, as well as tenderness and aerobic endurance after 12 weeks of LPA in minimally active adults with FM.

\section{Materials and methods Participants}

Participants were 92 adults ( 88 women and 4 men) aged 18 years or older who met American College of Rheumatology diagnostic criteria for FM [13]. The mean (SD) age of participants was $47.7 \pm 10.7$ years and $80 \%$ were white. The mean duration of FM was $7.5 \pm 6.2$ years. At enrollment, participants were not meeting the US Surgeon General's 1996 recommendation for physical activity [11] for the previous six months (that is, not engaging in either moderate-intensity physical activity for $\geq 30$ minutes on $\geq$ five days per week or vigorous physical activity $\geq$ three times per week for $\geq 20$ minutes each time during the previous month). Persons with acute or chronic medical conditions that could preclude active participation (for example, cancer, coronary artery disease) were excluded from the trial. We also excluded those who intended to change medications that might affect mood, those who intended to seek professional treatment for anxiety or depression during the study period, and those who were unwilling to make the required time commitment.

Participants were recruited from the Johns Hopkins Arthritis Center, affiliated Johns Hopkins Rheumatology clinics, by advertisements in the Arthritis Foundation Maryland Chapter newsletter, newspaper advertisements, and via clinical trial recruitment websites, including clinicaltrials.gov. All participants completed baseline testing which included a series of questionnaires, a tender point examination, and a six-minute walk test. At baseline, participants also wore a waist-mounted pedometer (AccuSplit Eagle 1020, Livermore, CA, USA) for seven days (recalibrating it each morning) and recorded their daily step count. These data were used to calculate the mean steps per day as an estimate of physical activity. This study was approved by the Institutional Review Board of Johns Hopkins University School of Medicine, and all participants gave written informed consent.

\section{Study procedures}

Participants were randomized via a coin flip at a 1:1 allocation ratio to each of the two groups. The group meetings for LPA and FME were held on different days to avoid contact between participants assigned to the different conditions. The interventions did not replace usual medical care and the participants had comparable durations of contact time with study staff (Table 1 summarizes the LPA and FME conditions).

\section{Lifestyle physical activity (LPA)}

Participants assigned to LPA attended six, 60-minute group sessions over 12 weeks. Delivered by one of the authors (KRF), the LPA protocol was identical to the one developed for our pilot study [12] and was loosely based on Active Living Every Day, a cognitive-behavioral physical activity promotion program developed by Dr. Steven Blair and colleagues at the Cooper Aerobics Center [14]. The LPA protocol addressed FM-specific challenges to becoming more physically active (that is, dealing with pain and fatigue, fear that physical activity will promote a flare) and discussed how LPA successfully addresses them. The goal of the LPA intervention was to increase moderate-intensity physical activity by helping participants find ways to accumulate short bouts of physical activity throughout the day. Participants were asked to gradually work their way up to meeting the Surgeon General's 1996 recommendation of accumulating 30 minutes, above usual activity, of moderateintensity LPA five to seven days each week.

At the first session, participants were taught how to perform their LPA at moderate-intensity (that is, you will be breathing heavily but not so heavily that you could not hold a conversation). They were also prescribed 15 minutes, above usual level, of accumulated moderate-intensity LPA five to seven days a week, and asked to increase the daily duration of LPA by five minutes each week. The five-minute increase in the daily duration of LPA was based on findings from our pilot study [12] and was well-tolerated by the majority of participants. Thus, by Week 5 , most participants were accumulating 30 minutes, above their usual level, of LPA five to seven days a week. Participants were free to accumulate more than 30 minutes of LPA five to seven days per week, if desired.

During subsequent sessions participants were taught self-monitoring of LPA, goal setting, dealing with 
Table 1 Description of lifestyle physical activity (LPA) and fibromyalgia education (FME) protocols*

\begin{tabular}{|c|c|c|}
\hline Component & LPA & FME \\
\hline Three, two-hour FM education and support meetings & $\mathrm{NO}$ & YES \\
\hline Physical activity intervention delivered in six, one-hour meetings & YES & NO \\
\hline Wear pedometer and keep a physical activity log & YES & NO \\
\hline Prescribed physical activity & YES & NO \\
\hline Approximately six hours of face-to-face contact time & YES & YES \\
\hline \multicolumn{3}{|l|}{ Topics Covered During the Meetings } \\
\hline LPA & FME & \\
\hline $\begin{array}{c}\text { "Physical Activity \& FM" } \\
\text { Described FM (symptoms, diagnosis, treatment)/benefits of physical activity/ } \\
\text { demonstrated moderate-intensity LE/prescribed LE and self-monitoring/identified and } \\
\text { addressed barriers to physical activity } \\
\text { (Sessions } 1 \& 2 \text { ) }\end{array}$ & $\begin{array}{l}\text { "FM: What is it and how is it diagnosed?" } \\
\text { Presented general information on the symptoms of } \\
\text { FM and how it is diagnosed; } \\
\text { Discussion and social gathering } \\
\text { (Session 1) }\end{array}$ & \\
\hline $\begin{array}{l}\text { "How to Keep Moving" } \\
\text { Discussed progress, effect on symptoms, goal setting, problem solving, importance of } \\
\text { self-monitoring, provided feedback, and troubleshooting } \\
\text { (Sessions } 3 \& 4 \text { ) }\end{array}$ & $\begin{array}{l}\text { "What causes FM?" } \\
\text { Presented the latest information on the causes and } \\
\text { consequences of FM; } \\
\text { Discussion and social gathering } \\
\text { (Session 2) }\end{array}$ & \\
\hline 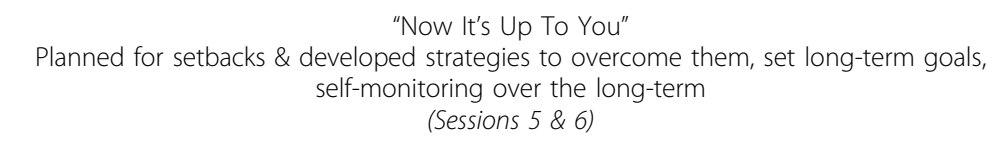 & $\begin{array}{l}\text { "Treating FM" } \\
\text { Discussed of medical and non-medical approaches, } \\
\text { including exercise, to treating FM; } \\
\text { Discussion and social gathering } \\
\text { (Session 3) }\end{array}$ & \\
\hline $\begin{array}{l}\qquad \text { LPA } \\
\text { Accumulate } \geq 30 \text { minutes of self-selected physical activity five to seven days per week }\end{array}$ & $\begin{array}{c}\text { FME } \\
\text { Did not alter their characteristic level of physical } \\
\text { activity }\end{array}$ & \\
\hline
\end{tabular}

*FM, fibromyalgia; FME = fibromyalgia education; LPA, lifestyle physical activity

symptom flares, problem solving strategies to overcome barriers to being more physically active, as well as instruction in finding new ways to integrate short bouts of LPA into their daily lives. Feedback focused on whether participants achieved the prescribed level of LPA, as well as the LPA's influence on symptoms.

Participants wore the waist-mounted pedometers to record their steps each day (that is, as an assessment of adherence to LPA). Participants were shown how to use the pedometer, where to place it, and how to record their steps on a step diary form. At the end of each day they recorded their steps on a diary form and zeroed their pedometer so they could record their steps for the next day. They also kept a diary that categorized the types of LPA's they engaged in (for example, garden/ outdoor activity, household activity, leisure activity). The step count data and diary entries were collected at each intervention session.

\section{Fibromyalgia education (FME)}

Participants assigned to the FME group met monthly for three months. FME was a minimal intervention with each session divided into three components: (1) education (45 to 60 minutes), (2) question and answer (20 to 30 minutes), and (3) social support (20 to 30 minutes). Conducted by an experienced FM support group facilitator, these 90- to 120-minute sessions presented information on the symptoms, diagnosis, and treatment of
FM. The rationale for FME was to provide education and to control for the effects of being enrolled in a clinical trial and receiving increased attention and social support. Moreover, by providing a minimal intervention, as opposed to a standard care control, we anticipated enhancing retention. The final session of FME presented information on exercise and physical activity, but no specific recommendations or prescription concerning exercise was given. To avoid the possibility that wearing a pedometer would increase their physical activity, FME participants only wore one for the baseline and posttesting assessments.

\section{Outcomes measures}

The following outcome measures were collected at baseline and after the 12-week intervention.

\section{Primary outcome \\ Perceived physical function}

Perceived physical function was assessed using the Fibromyalgia Impact Questionnaire (FIQ) total score. The FIQ is a well-validated 10-item questionnaire that measures aspects of physical functioning in patients with FM [15]. The FIQ is scored so that higher scores are indicative of poorer functioning. Test-retest reliability ranged from .56 to .95 and construct validity relative to tender points was acceptable ( $\mathrm{rs}=$ approximately .56$)$ [15]. 


\section{Secondary outcomes \\ Pain}

Pain was assessed using a $100 \mathrm{~mm}$ Visual Analogue Scale (VAS) where participants rated their current level of pain, ranging from 0 (no pain) to 100 (worse pain imaginable).

\section{Fatigue}

The Fatigue Severity Scale (FSS) [16] was used to assess the current level of fatigue. The FSS is a nine-item questionnaire, answered on a seven-point scale, ranging from strongly agree to strongly disagree. The FSS has good internal consistency (Cronbach's alpha $=.81$ ) and correlates with VAS fatigue measures $(r=.68)$ [16].

\section{Depression}

Depression was assessed using the Center for Epidemiologic Studies Depression Scale (CES-D) [17]. The CES-D contains 20-items rated on a four-point Likert scale ranging from 0 (rarely or none of the time) to 3 (most or all of the time), and measures symptoms during the past week. The CES-D is a widely used measure of depressive symptoms and has acceptable internal consistency $(.84$ to .90$)$ and validity $(r=.56$ with clinical rating of depression severity) [17].

\section{Tenderness}

A digital tender point examination, at the 18 sites specified in the American College of Rheumatology FM classification criteria [13], was completed at baseline and after the intervention. Tender point counts are moderately reliable in classifying the tenderness associated with FM (kappa $=.75$ ) and inter-rater agreement on the presence of tenderness through digital examination is .51 [13].

\section{Body mass index (BMI: $\mathrm{kg} / \mathrm{m}^{2}$ )}

Weight and height were recorded at each assessment and these variables were used to calculate BMI, an index of body weight adjusted for height.

\section{Six-minute walk test}

The six-minute walk [18] is a measure of aerobic endurance. For this test, participants walked as far they could in six minutes on a preselected course, with the distance walked recorded. The reliability of the six-minute walk test is excellent $(r=.91)$ and it correlates with the FIQ $(\mathrm{r}=-.49)$ and is sensitive to change due to exercise in distance walked $(+78 \mathrm{~m})$, and $\mathrm{VO}_{2}(+1.8 \mathrm{ml} / \mathrm{kg} / \mathrm{min})$ [18]. The six-minute walk test was measured at baseline and at post-testing. We expressed the results as meters per second, an index of gait speed.

\section{Sample size and data analysis}

Thirty-five adults with FM per group were projected to provide a power of $80 \%$ to detect a clinically significant $20 \%$ difference between the groups on the FIQ score. Ninety-two participants were enrolled to allow for a $25 \%$ post-randomization drop out rate.
Baseline data between the two groups were compared using t-tests or Chi Square tests. Changes in perceived physical function, depression, pain, tenderness, fatigue, $B M I$, and the six-minute walk test were compared between the LPA and FME groups using between-subjects t-tests. Because there was a significant difference between the LPA and FME groups on self-reported duration of FM (see Table 2), as a sensitivity analysis, we adjusted scores of the outcome measures for the duration of FM and replicated the analyses. We also used regression techniques to adjust the outcome measures on the basis of whether or not the participant reported any change in their ongoing FM treatments, either pharmacologic or non-pharmacologic $(0=$ no change, 1 = change) during the trial. Because the results did not differ as a function of these adjustments, we present the results for the unadjusted outcome variables. Although data from all subjects were analyzed regardless of whether those subjects complied with or remained in treatment, participants with missing data on a particular variable were excluded from that particular analysis. We also performed an analysis among participants who completed the 12-week trial (completers only). Cohen's $d$ effects size estimates [19] were calculated for each difference on the outcome measures between LPA and FME. Analyses were performed using SPSS software, Version 16. Two-tailed $P$ values of $<0.05$ were used to denote statistical significance.

\section{Results}

Nine participants withdrew after baseline testing but prior to randomization (see Figure 1). We randomized 46 participants into the LPA intervention and 38 into the FME group in five separate cohorts of approximately 8 to 10 per cohort at six-month intervals. (Because the FME facilitator was unavailable, one smaller cohort $(\mathrm{N}=4)$ was comprised of only LPA participants). Selected baseline characteristics of the 84 participants are shown in Table 2. With the exception of duration of FM, the two groups were comparable on age, race, education, employment status, BMI, and the use of medications for FM or for other medical conditions.

Seventy-three of the 84 participants (87\%) completed the 12-week intervention and post-testing. Drop outs were unrelated to randomized treatment assignment $(P=.988)$ and there were no significant differences on any baseline variables between those who dropped out and those who completed post-testing. There was also no difference in the mean percentage of meetings attended by those randomized to the FME (77\%) and LPA $(72 \%)$ groups $(P=.542)$.

As shown in Figure 2, the LPA group significantly increased the mean number of daily steps from 3,788 \pm 2,135 at baseline to $5,837 \pm 1,770$ at the final 
Table 2 Baseline characteristics of the randomized participants*

\begin{tabular}{|c|c|c|c|}
\hline Characteristic & Lifestyle Physical Activity (LPA) & $\begin{array}{l}\text { Fibromyalgia Education } \\
\text { (FME) }\end{array}$ & $P$ value \\
\hline $\mathrm{N}(\%)$ of participants & $46(55)$ & $38(45)$ & \\
\hline Age, years & $46.4 \pm 11.6$ & $49.0 \pm 10.2$ & 0.287 \\
\hline Female, N (\%) & $43(94)$ & $38(100)$ & 0.248 \\
\hline Self-reported race, N (\%) & & & 0.789 \\
\hline White & $36(78)$ & $31(82)$ & \\
\hline Non-White & $10(22)$ & $7(18)$ & \\
\hline Marital status, N (\%) & & & 0.519 \\
\hline Married or cohabitating & $24(52)$ & $24(63)$ & \\
\hline Widowed, divorced, or separated & $12(27)$ & $11(29)$ & \\
\hline Single & $10(22)$ & $3(8)$ & \\
\hline Educational level, N (\%) & & & 0.454 \\
\hline Postgraduate & $9(20)$ & $5(13)$ & \\
\hline College graduate & $16(34)$ & $11(32)$ & \\
\hline Some college & $11(24)$ & $13(34)$ & \\
\hline High school & $10(22)$ & $8(21)$ & \\
\hline Employment status, N (\%) & & & 0.923 \\
\hline Employed & $20(43)$ & $18(47)$ & \\
\hline Unemployed or Disabled & $11(24)$ & $9(24)$ & \\
\hline Retired or Other & $15(33)$ & $11(29)$ & \\
\hline Years since diagnosis ${ }^{a}$ & $5.9 \pm 5.1$ & $9.6 \pm 6.8$ & 0.007 \\
\hline Steps per day & $3,788 \pm 2,135$ & $3,071 \pm 1,810$ & 0.139 \\
\hline Taking FM medications, N (\%) ${ }^{a}$ & $31(82)$ & $40(87)$ & 0.498 \\
\hline Taking other medications, N (\%) ${ }^{\mathrm{a}}$ & $33(87)$ & $44(96)$ & 0.146 \\
\hline
\end{tabular}

*Data for categorical variables are presented as $\mathrm{N}^{\prime}$ and percentages; data for continuous variables are presented as means \pm standard deviation. a Obtained from self-report.

FME, fibromyalgia education; LPA, lifestyle physical activity

intervention session $(P=.001)$. This represents a $54 \%$ increase in the mean number of daily steps over the course of the 12-week intervention. Although walking was the most common form of LPA, other popular forms of LPA included garden/outdoor activity (for example, mowing the lawn, planting flowers, pulling weeds); household activity (for example, cleaning out a closet, vacuuming, doing laundry); and sports activity (for example, cycling, swimming, field hockey).

At baseline, there were no significant differences between the LPA and FME groups on FIQ, pain, fatigue, depression, number of tender points, BMI, and six-minute walk distance (see Table 3). At post-testing, compared to the FME group, the LPA group reported significant reductions in the FIQ score $(P=.032$; Cohen's $d=.53)$ and in pain $(P=.006$; Cohen's $d=.67)$. The difference between the LPA and FME groups on the sixminute walk test approached significance $(P=.067$; Cohen's $d=.53$ ). There were no significant differences between the groups on BMI, fatigue, depression, or the number of tender points. The results (data not shown) were not materially altered when the analysis was restricted to only participants who completed the entire 12-week trial (that is, completers only analysis).

\section{Discussion}

The 12-week program, designed to help minimally active adults with FM increase their physical activity by working toward accumulating at least 30 minutes of self-selected moderate-intensity physical activity most days of the week, produced a $54 \%$ increase in the average number of steps taken per day. Compared to the FME control group, LPA participants significantly reduced their perceived functional deficits (that is, FIQ score) and pain. Moreover, compared to FME, the LPA participants had a greater improvement on the six-minute walk (expressed as gait speed), although this difference failed to reach statistical significance. The magnitude of the post-intervention differences, expressed as percent change from LPA to FME groups, were $18 \%$ for the FIQ score and $35 \%$ for the pain VAS score. When expressed as Cohen's $d$ effect sizes these are indicative of medium-sized effects. Moreover, the change on the FIQ score exceeds the minimally clinically important difference of $14 \%$ recently identified [20], suggesting that increasing physical activity via LPA produces changes on perceived physical function that are of a relevant magnitude. On the other hand, the effect of LPA on the six-minute walk test was not 


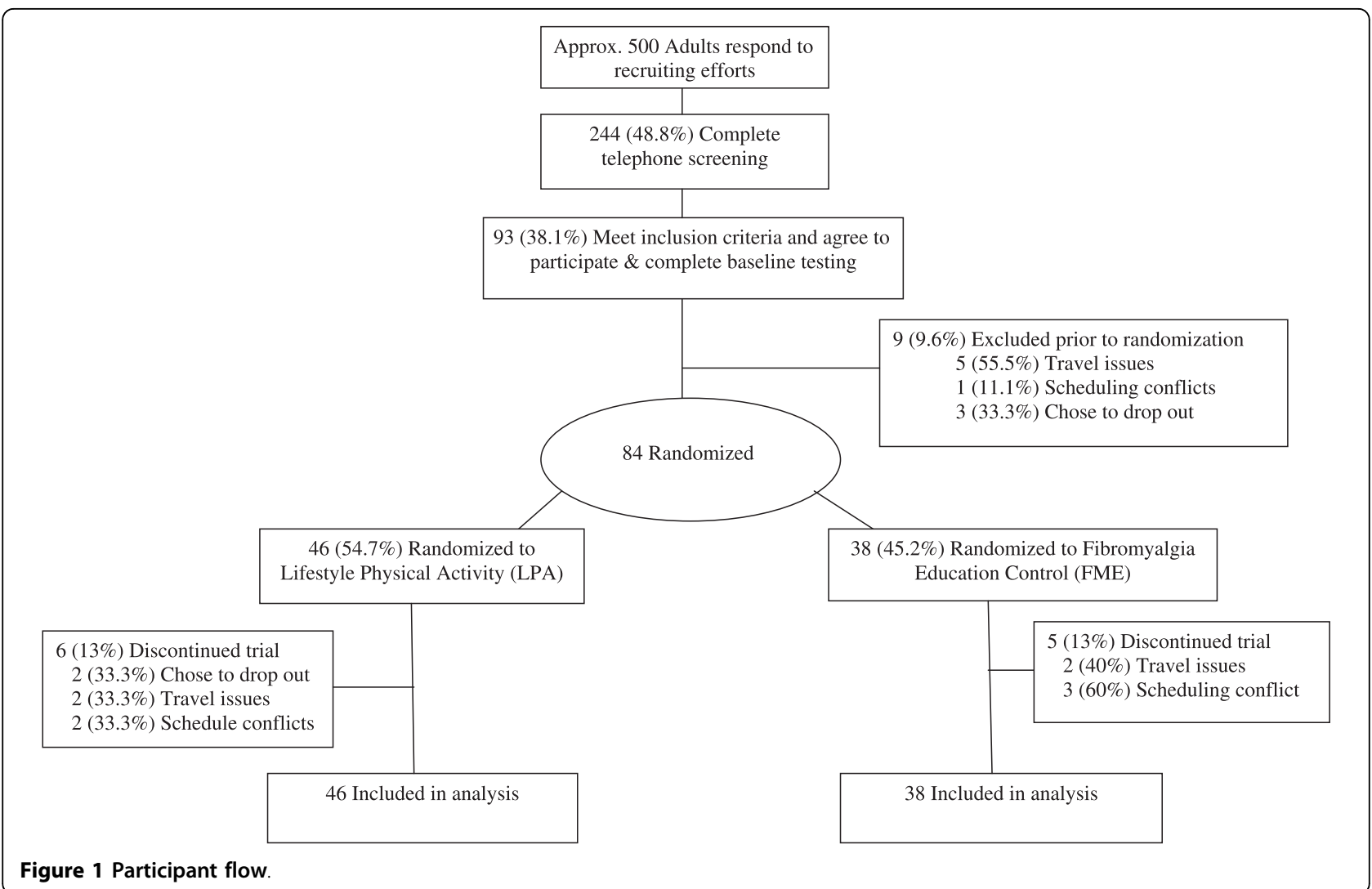

Figure 1 Participant flow.

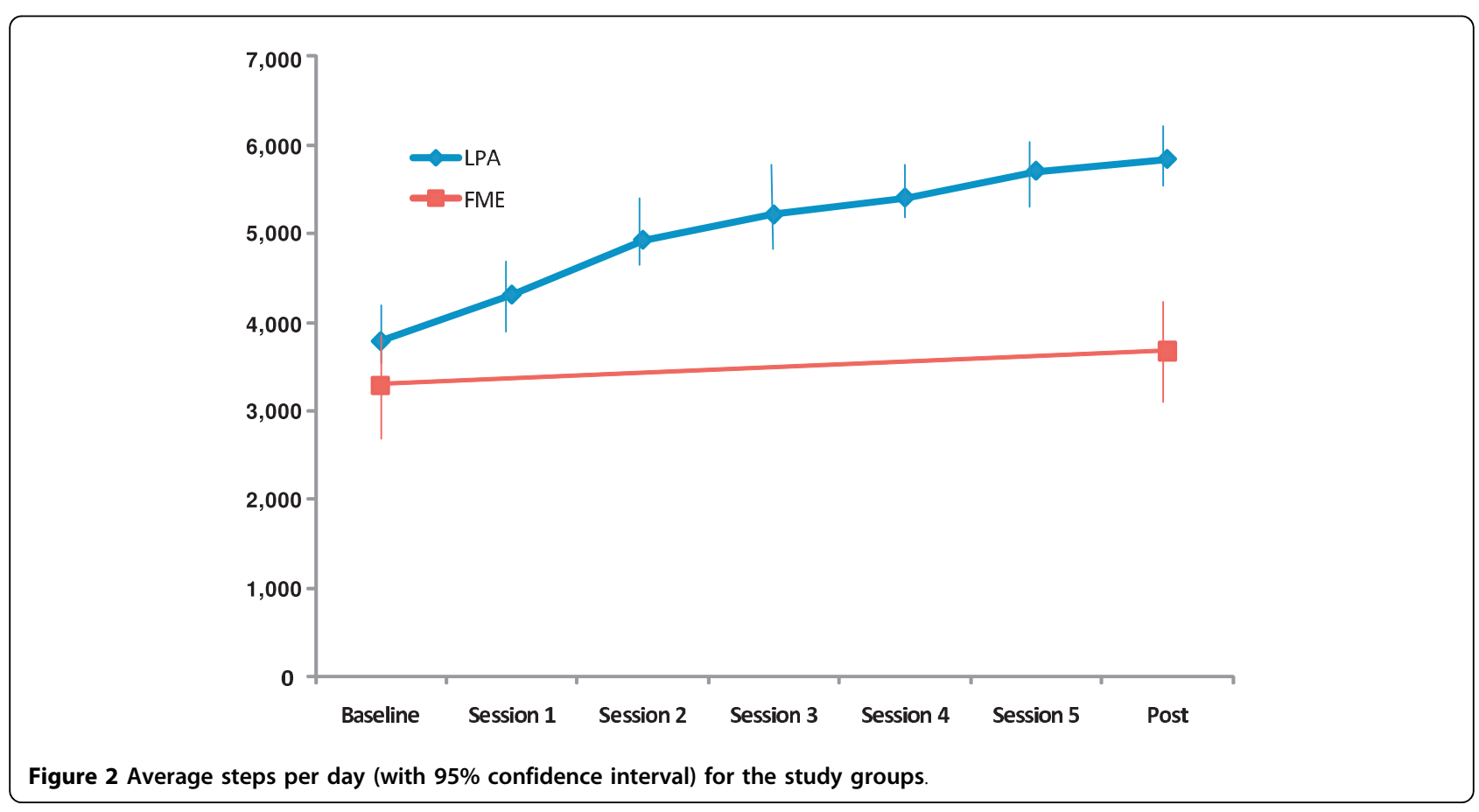


Table 3 Differences between lifestyle physical activity (LPA) and fibromyalgia education (FME) groups on the primary and secondary study measures ${ }^{a}$

\begin{tabular}{|c|c|c|c|c|c|c|}
\hline \multirow[t]{2}{*}{ Variable } & \multicolumn{3}{|c|}{ Mean \pm SD } & \multirow{2}{*}{$\begin{array}{l}\text { Mean difference between groups at baseline and at 12-weeks } \\
\qquad(95 \% \mathrm{Cl})\end{array}$} & \multirow{2}{*}{$\begin{array}{c}P \\
\text { Value }\end{array}$} & \multirow{2}{*}{$\begin{array}{c}\text { Cohen's } \\
d\end{array}$} \\
\hline & $\bar{N}$ & LPA & FME & & & \\
\hline \multicolumn{7}{|l|}{ FM impact questionnaire } \\
\hline Baseline & 84 & $\begin{array}{c}67.5 \pm \\
12.0\end{array}$ & $\begin{array}{c}69.7 \pm \\
13.4\end{array}$ & $2.2(-3.3$ to 7.8$)$ & 0.424 & .17 \\
\hline Post intervention & 73 & $\begin{array}{c}56.7 \pm \\
20.6\end{array}$ & $\begin{array}{c}67.0 \pm \\
18.6\end{array}$ & $10.2(.91$ to 19.6$)$ & 0.032 & .53 \\
\hline \multicolumn{7}{|l|}{ Pain } \\
\hline Baseline & 84 & $\begin{array}{c}54.6 \pm \\
25.6\end{array}$ & $\begin{array}{c}58.9 \pm \\
25.0\end{array}$ & $4.3(-7.2$ to 14.9$)$ & 0.489 & .17 \\
\hline Post intervention & 73 & $\begin{array}{c}46.3 \pm \\
24.2\end{array}$ & $\begin{array}{c}62.4 \pm \\
24.5\end{array}$ & 16.1 (4.6 to 27.5$)$ & 0.006 & .67 \\
\hline \multicolumn{7}{|l|}{ Fatigue severity scale } \\
\hline Baseline & 84 & $51.9 \pm 9.3$ & $52.3 \pm 9.1$ & $.4(-3.6$ to 4.4$)$ & 0.843 & .04 \\
\hline Post intervention & 73 & $50.6 \pm 9.9$ & $\begin{array}{c}51.4 \pm \\
10.1\end{array}$ & $.8(-3.9$ to 5.5$)$ & 0.727 & .07 \\
\hline \multicolumn{7}{|l|}{ CES-D* } \\
\hline Baseline & 84 & $23.4 \pm 8.6$ & $24.0 \pm 10$ & $.6(-3.8$ to 4.5$)$ & 0.798 & .06 \\
\hline Post intervention & 73 & $21.6 \pm 9.8$ & $\begin{array}{c}21.2 \pm \\
11.3\end{array}$ & $.4(-5.3$ to 4.6$)$ & 0.888 & .04 \\
\hline \multicolumn{7}{|c|}{ Number of tender points } \\
\hline Baseline & 84 & $16.2 \pm 2.3$ & $16.1 \pm 3.2$ & $.1(-1.2$ to 1.0$)$ & 0.979 & .03 \\
\hline Post intervention & 72 & $16.0 \pm 2.3$ & $16.8 \pm 2.0$ & $.8(-.35$ to 1.9$)$ & 0.172 & .37 \\
\hline \multicolumn{7}{|l|}{ Body mass index (BMI) } \\
\hline Baseline & 82 & $31.4 \pm 8.4$ & $29.8 \pm 6.2$ & $1.6(-4.7$ to 1.7$)$ & 0.360 & .22 \\
\hline Post intervention & 60 & $31.0 \pm 9.0$ & $29.9 \pm 6.2$ & $1.1(-5.3$ to 2.9$)$ & 0.575 & .14 \\
\hline \multicolumn{7}{|l|}{$\begin{array}{l}\text { Six-minute walk test, yd/ } \\
\text { sec }\end{array}$} \\
\hline Baseline & 77 & $\begin{array}{c}1.08 \pm \\
0.15\end{array}$ & $\begin{array}{c}1.08 \pm \\
0.19\end{array}$ & $.0004(-0.78$ to 0.79$)$ & 0.991 & 0 \\
\hline Post intervention & 62 & $\begin{array}{c}1.24 \pm \\
0.28\end{array}$ & $\begin{array}{c}1.11 \pm \\
0.20\end{array}$ & $1.21(-0.25$ to 0.008$)$ & 0.067 & .53 \\
\hline
\end{tabular}

${ }^{*}$ Center for Epidemiologic Studies Depression Scale; ${ }^{\text {a }}$ between-subjects t-tests were used to derive P values; ${ }^{\text {b }}$ Cohen's $d$ [19] effect size estimates ( $d=.20$ (small effect); $d=.50$ (medium effect); $d=.80$ (large effect)).

FME, fibromyalgia education; LPA, lifestyle physical activity

statistically significant (although it produced a Cohen's $d$ of .53). It is important to note that there was a smaller sample size available for this analysis which reduced statistical power.

In general our results are in accord with studies investigating the effects of exercise on people with FM $[7,8,21,22]$. Specifically, the majority of studies suggest that exercise can produce mild-to-moderate benefits on aerobic endurance, strength, functional status, and quality of life $[7,23,24]$. However, because the exercise interventions investigated vary so markedly in type (for example, water aerobics, traditional aerobics, T'ai Chi, strength training), frequency, intensity, and duration it is difficult to compare results across studies.

One thing seems clear from the FM exercise literature, people with FM have difficulty adhering to exercise. Indeed, in FM clinical exercise trials drop-out rates often nearly exceed $30 \%$ [for example, $[8,24]]$ suggesting that developing exercise interventions that can be sustained is perhaps as important a goal as finding the particular interventions that produce optimal benefits.

The magnitude of the effects of LPA observed in this study on perceived physical function and pain were similar to those obtained in our smaller pilot study [12]. These effects were also generally consistent with other protocols that involve low-to-moderate intensity exercise, interventions that appear to produce the greatest level of compliance in people with FM [for example, $[7,8,24]]$.

It is important to note that even though the LPA group increased their mean daily steps by $54 \%$, it only moved them, as defined by the pedometer-determined physical activity classifications developed by TudorLocke and colleagues [25], from the sedentary $(<5,000$ 
steps/day) to the low active (5,000 to 7,499 steps/day) category. Indeed, the mean steps per day at post-testing among the LPA participants were comparable to the mean daily steps observed in patients with progressive neuromuscular disease, and are significantly lower than other special populations such as diabetics, patients undergoing breast cancer treatment, and those with joint replacements [26]. This suggests that, even with the initiation of LPA, people with FM progress only to a relatively low level of physical activity. It is important to note, however, that the trajectory of the mean step count continued to rise over the 12 weeks suggesting that, had the trial continued, their physical activity may have continued to increase. It may be that people with FM require more time to eventually reach physical activity recommendations compared to persons with other chronic conditions.

This study has limitations and strengths. First, to minimize attrition and control for the effects of increased attention, participants randomized to the FME group did receive a minimal intervention. Thus, we cannot determine how LPA compares with a traditional no treatment control group. Second, with the exception of BMI, the tender point count and six-minute walk test, the outcomes described herein were derived from selfreport and may be influenced by a variety of factors, including those associated with enrollment in a clinical trial. Third, using pedometers to assess LPA is relatively crude and does not quantify other sorts of physical activities that participants may have engaged in such as cycling or water exercise. Fourth, we did not measure muscle strength during the trial so we are unable to determine whether LPA influences strength. Finally, we excluded persons with FM who had other co-morbid conditions such as uncontrolled hypertension or arthritis which may limit the generalizability of our findings. Strengths of this study include the randomized design, a relatively small drop-out rate (13\%), the LPA group's adherence to standardized intervention protocol, and the relatively high rates of attendance to the group sessions.

\section{Conclusions}

The results of this study suggest that promoting increased physical activity by asking persons with FM to accumulate short bouts of activity throughout the day can markedly increase the average number of steps taken per day and produces clinically relevant reductions in perceived functional deficits and pain. However, the LPA intervention only moved the participants from the sedentary to low physical activity category. This suggests that it is essential to encourage FM patients to increase the duration of their physical activity in ways that do not compromise their ability to sustain the increased level of activity over the intermediate- and long-term.

\section{Abbreviations}

BMI: Body Mass Index; CES-D: Center for Epidemiologic Studies Depression Scale; FIQ: Fibromyalgia Impact Questionnaire; FM: fibromyalgia; FME: Fibromyalgia Education Control Group; FSS: Fatigue Severity Scale; LPA: lifestyle physical activity; SD: standard deviation; VAS: Visual Analogue Scale.

\section{Acknowledgements}

Grant support: NIH/NIAMS grant AR053168. The funding body played no role in the study design; in the collection, analysis, and interpretation of data; in the writing of the manuscript; and in the decision to submit the manuscript for publication. We also acknowledge the support of the Johns Hopkins Bayview Clinical Research Unit for assistance with data collection.

\section{Author details}

'Division of Rheumatology, Johns Hopkins University School of Medicine, 5200 Eastern Avenue, Baltimore, MD 21224, USA. Departments of Anesthesiology and Medicine (Rheumatology), University of Michigan, Chronic Pain \& Fatigue Research Center, 24 Frank Lloyd Wright Lobby M, Ann Arbor, MI 48106, USA.

\section{Authors' contributions}

KF conceived of the study, acquired the funding, participated in the design of the study, the delivery of the intervention, performed the statistical analysis, and drafted the manuscript. LC carried out the recruitment, enrollment, and data collection. DC participated in designing the study and assisted with the drafting of the manuscript. All authors read and approved the final manuscript.

\section{Competing interests}

Kevin R. Fontaine and Lora Conn declare that they have no competing interests. Daniel J. Clauw has acted as a consultant for Pfizer, Lilly, Forest Laboratories, Cypress Biosciences, Pierre Fabre, UCB, and Wyeth, and has received grant support from Pfizer, Cypress Bioscience, and Forest.

Received: 26 October 2009 Revised: 4 February 2010 Accepted: 30 March 2010 Published: 30 March 2010

\section{References}

1. Perrot S, Dickenson AH, Bennett RM: Fibromyalgia: Harmonizing science with clinical practice considerations. Pain Pract 2008, 8:177-189.

2. Lawrence RC, Felson DT, Helmick CG, Arnold LM, Choi H, Deyo RA, Gabriel S, Hirsch R, Hochberg MC, Hunder GG, Jordan JM, Katz JN, Kremers HM, Wolfe F: Estimates of the Prevalence of Arthritis and Other Rheumatic Conditions in the United States Part II. Arthritis Rheum 2008, 58:26-35.

3. Marcus DA: Fibromyalgia diagnosis and treatment options. Gend Med 2009, Suppl 2:139-151.

4. Williams DA, Clauw DJ: Understanding fibromyalgia: Lessons from the broader pain research community. J Pain 2009, 10:777-791.

5. Goldenberg DL, Burckhardt C, Crofford L: Management of fibromyalgia syndrome. JAMA 2004, 292:2388-2385.

6. Gore M, Sadosky AB, Zlatera G, Clauw DJ: Clinical characteristics, pharmacotherapy and healthcare resource use amongpatients with fibromyalgia newly prescribed gabapentin or pregabalin. Pain Pract 2009, 9:363-374

7. Busch AJ, Schacter CL, Overend TJ, Peloso PM, Barber A: Exercise for fibromyalgia: a systematic review. J Rheum 2008, 35:1130-1144.

8. Jones KD, Liptan GL: Exercise Interventions in Fibromyalgia: Clinical Applications from the Evidence. Rheum Dis Clin North Am 2009, 35:373-391.

9. Dunn AL, Marcus BH, Kampert JB, Garcia ME, Kohl HW III, Blair SN: Comparison of lifestyle and structured interventions to increase physical activity and cardiorespiratory fitness. JAMA 1999, 281:327-334.

10. Plonczynski DJ, Wilbur JE, Larson JL, Keith TK: Lifestyle Physical Activity of Older Rural Women. Res Nurs Health 2008, 31:501-513. 
11. U.S. Department of Health and Human Services: Physical Activity and Health: A Report of the Surgeon General Centers for Disease Control and Prevention (CDC), National Center for Chronic Disease Prevention and Health Promotion, Atlanta 1996.

12. Fontaine KR, Haaz S: Effects of lifestyle physical activity on health status, pain, and function in adults with fibromyalgia syndrome. J Musculoskel Pain 2007, 15:3-9.

13. Wolfe F, Smythe HA, Yunus MB, Bennett RM, Bombardier C, Goldenberg DL, Tugwell P, Campbell SM, Abeles M, Clark P, Fam AG, Farber SJ, Fiechtner JJ, Franklin CM, Gatter RA, Hamaty D, Lessard J, Lichbroun AS, Masi AT, Mccain GA, Reynolds WJ, Romano TJ, Russell IJ, Sheon RP: The American College of Rheumatology 1990 Criteria for the Classification of Fibromyalgia. Report of the Multicenter Criteria Committee. Arthritis Rheum 1990, 33:160-172.

14. Blair SN, Dunn AL, Marcus BH, Carpenter RN, Jaret P: Active Living Every Day Champaign: Human Kinetics 2002.

15. Burckhardt CS, Clark SR, Bennett RM: The fibromyalgia impact questionnaire: Development and validation. J Rheum 1991, 18:728-733.

16. Krupp LB, LaRocca NG, Muir-Nash J, Steinberg AD: The fatigue severity scale: application to patients with multiple sclerosis and systemic lupus erythematosus. Arch Neurol 1989, 46:1121-23.

17. Radloff LS: The CES-D Scale: a self-report depression scale for research in the general population. Appl Psychol Meas 1977, 1:385-401.

18. Pankoff $B$, Overend T, Lucy D, White $K$ : Validity and responsiveness of the 6 minute walk test for people with fibromyalgia. J Rheum 2000, 27:2666-2670.

19. Cohen J: Statistical Power Analysis for the Behavioral Sciences Hillsdale: Erlbaum, 21988.

20. Bennett RM, Bushmakin AG, Cappelleri JC, Zlateva G, Sadosky AB: Minimally clinically important difference in the Fibromyalgia Impact Questionnaire. J Rheum 2009, 36:1304-1311.

21. Goldenberg DL: Multidimensional modalities in the treatment of fibromyalgia. J Clin Psychiatry 2008, 69:30-34.

22. Rooks DS, Gautim S, Romeling M, Cross ML, Stratigakis D, Evans B, Goldenberg DL, Iversen MD, Katz JN: Group exercise, education, and combination self-management in women with fibromyalgia. Arch Intern Med 2007, 167:2192-2200.

23. Nørregaard J, Lykkegaard J, Mehlsen J, Danneskiold-Samsøe B: Exercise training in treatment of fibromyalgia. J Musculoskel Pain 1997, 5:71-79.

24. Jones KD, Burckhardt CS, Clark SR, Bennett RM, Potempa KM: A randomized controlled trial of muscle strengthening versus flexibility training in fibromyalgia. J Rheumatol 2002, 29:1041-1048.

25. Tudor-Locke $C$, Hatano Y, Pangrazi RP, Kang M: Revisiting "how many steps are enough?" Med Sci Sports Exerc 2008, 40:S537-543.

26. Tudor-Locke C, Washington TL, Hart TL: Expected values for steps per day in special populations. Prev Med 2009, 49:3-11.

doi:10.1186/ar2967

Cite this article as: Fontaine et al:: Effects of lifestyle physical activity on perceived symptoms and physical function in adults with fibromyalgia: results of a randomized trial. Arthritis Research \& Therapy 2010 12:R55.

\section{Submit your next manuscript to BioMed Central and take full advantage of:}

- Convenient online submission

- Thorough peer review

- No space constraints or color figure charges

- Immediate publication on acceptance

- Inclusion in PubMed, CAS, Scopus and Google Scholar

- Research which is freely available for redistribution

Submit your manuscript at www.biomedcentral.com/submit
C Biomed Central 